\title{
Trasplante renal en bloque de donantes pediátricos
}

\author{
Pedro Méndez-Chacón ${ }^{1,2}$, Armando Vidalón ${ }^{1,2}$, Carmen Berríos ${ }^{2}$, Miguel Camacho ${ }^{2}$
}

Resumen

Objetivo: Determinar la aceptación de ambos riñones de donantes pediátricos para utilizarlos como trasplante renal en bloque. Material y Métodos: La edad de los seis donantes varió entre 14 meses y 10 años. La causa de muerte fue el traumatismo encefalocraneano. La edad de los seis receptores osciló entre 15 y 48 años con peso menor de $50 \mathrm{~kg}$. Ambos riñones fueron removidos en bloque. La técnica operatoria de los trasplantes consistió en la anastomosis término lateral del segmento aórtico y vena cava inferior del donante a los vasos ilíacos del receptor; ambos uréteres fueron anastomosados por separado a la vejiga. El implante fue localizado en una de las fosas ilíacas. Resultados: Los injertos en bloque funcionaron inmediatamente. Cuatro de los seis receptores cursaron con función renal adecuada a cuatro años promedio de seguimiento. Uno de los injertos en bloque se perdió por rechazo vascular severo y otro por indisciplina a la medicación inmunosupresora. Las series ecográficas de los riñones revelaron incremento de tamaño hasta en 55\% de su dimensión original al tercer mes del trasplante. Conclusión: Se recomienda que los riñones procedentes de donantes menores de tres años de edad sean utilizados como trasplante en bloque y riñones de donantes mayores de tres años como trasplante renal simple. Se demuestra que es posible trasplantar exitosamente riñones en bloque de niños muy pequeños en receptores mayores, según experiencia inédita en nuestro medio.

Palabras clave Trasplante de riñón; donandores de tejido; niño hospitalizado; riñón.

\section{Block kidney transplantation from pediatric donors}

\section{Abstract}

Objective: To determine both kidneys from pediatric donors acceptance in block kidney transplantation. Material and Methods: Age of the six donors ranged form 14 months to 10 year-old. The death cause was cranial trauma. Receptors' ages ranged between 15 and 48 years with weight less than $50 \mathrm{~kg}$. Both kidneys were removed in block. The transplants surgical technique consisted of both terminal-lateral anastomosis of the donor's aortic segment and inferior cava vein anastomosis to receptor's iliac vessels; both ureters were separately anastomosed to the bladder. The allograph was located in one iliac fossa. Results: The block allograph worked immediately. Four of six receptors kept adequate renal function in an average of four years surveillance time. One of the block's allograph

\footnotetext{
1 Universidad Nacional Mayor de San Marcos. Lima, Perú.

2 Servicio Nefrología, Hospital Nacional Edgardo Martins. Lima, Perú.
}

was lost because of severe vascular rejection and another because of indiscipline in the use of immunosuppressive medication. Kidney's ultrasonographic series revealed size increase up to $55 \%$ by the third month of transplantation. Conclusion: We suggest that kidneys from donors less than 3 year-old should be used as block transplant and kidneys coming from older donors should be used as simple kidney transplants. Our novel experience suggests that it is possible to successfully transplant kidneys in block from very little children to older receptors.

Key words: Kidney transplantation; tissue donors; child, hospitalized; kidney.

\section{INTRODUCCIÓN}

La lista de espera para trasplante renal continúa en ascenso (30\% por año), mientras el número de trasplantes permanece estacionario en el país. La escasez de donantes se constituye en serio problema para nuestra actividad trasplantadora $\left({ }^{1-3}\right)$. Las organizaciones de 
transplante se ven presionadas a incrementar la cantidad pero sin mermar la calidad de los trasplantes, recurriendo a los llamados donantes marginales.

Los centros de trasplante reportan cada vez con mayor frecuencia la utilización de donantes a corazón parado $\left(^{3,4}\right)$, infectados con virus de la hepatitis $C\left({ }^{3,5}\right)$, añosos $\left(^{3}\right)$, pediátricos $\left({ }^{1,3,6-}\right.$ $\left.{ }^{10}\right)$ y aún anencefálicos $\left({ }^{11-13}\right)$, todos ellos catalogados como donantes subóptimos.

El donante pediátrico cadavérico como fuente de órganos es de uso controversial $\left({ }^{14,15}\right)$, siendo en nuestro país subutilizado. Sólo $13 \%$ de los donantes correspondió a donantes menores de 10 años. Su uso se asocia con altas tasas de complicaciones técnicas, trombosis del injerto, rechazo temprano e injuria por hiperfiltración $\left({ }^{14,15}\right)$. Aminorar las dificultades quirúrgicas ocasionadas por los vasos y uréteres de pequeño calibre $\left({ }^{16}\right)$ es el reto que tienen los centros trasplantadores cuando se enfrentan a donantes pediátricos. Una alternativa constituye el trasplante renal en bloque y con resultados alentadores $\left({ }^{15,17}\right)$. En esta comunicación reportamos nuestra experiencia de trasplante renal en bloque con donante pediátrico por ser alternativa viable con alto porcentaje de sobrevida del injerto y nueva esperanza para los pacientes en lista de espera.

\section{MATERIAL Y MÉTODOS}

La edad de los seis donantes varió entre 14 meses y 10 años, con pesos de 10 a $22 \mathrm{~kg}$. La causa de muerte de todos ellos fue el traumatismo encefalocraneano.

La edad de los receptores osciló entre los 13 y 48 años y el peso en todos ellos fue menor a 50 kilos (Tabla 1). Se excluyó los pacientes de riesgo inmunológico alto o riesgo de complicaciones médicas.

Los riñones fueron removidos en bloque y preservados en solución de Eurocollins.
La técnica operatoria de los trasplantes renales en bloque incluyó la anastomosis termino lateral del segmento aórtico y vena cava inferior del donante a los vasos ilíacos externos del receptor único y los uréteres anastomosados por separado a la vejiga. El implante fue localizado en una de las fosas ilíacas. El tiempo de isquemia fría varió de 15 a 27 horas y el esquema inmunosupresor se basó en ciclosporina A, micofenolato mofetil y prednisona.

\section{RESULTADOS}

Los injertos en bloque funcionaron inmediatamente después del trasplante. En la evolución, dos de los seis trasplantes en bloque se perdieron, uno por trombosis venosa postepisodio de rechazo vascular severo y el otro por indisciplina en la medicación inmunosupresora. Un tercer receptor perdió uno de los riñones del injerto en bloque por estenosis ureteral, en tanto el riñón remanente mantuvo adecuada función renal (Tabla 2). Las series ecográficas de los riñones revelaron incremento del tamaño hasta en $55 \%$ de su dimensión original al tercer mes del trasplante (Tabla 3).

\section{DISCUSIÓN}

Los donantes marginales constituyen solución viable a la carencia de órganos $\left({ }^{4,10}\right)$. El donante pediátrico es una fuente subutilizada y puede representar importante contribución a la fuente de donantes, no obstante existir grupos trasplantadores renuentes a utilizar esta variedad de donación de riñones. La mayoría de centros del área de Eurotrasplant (49 de 68) no aceptan riñones de donante pediátrico ${ }^{8}$ ). Sólo $19 \%$ de los 184 centros de trasplante renal de Estados Unidos de Norteamérica aceptan donantes menores de dos años de edad y anencefálicos $\left({ }^{11,18}\right)$. La literatura médica describe alta incidencia de complicaciones vasculares y urológicas ocasionada en parte por el tamaño reducido de sus estructuras vasculares y 
Tabla 1. Características de los donantes y receptores del trasplante renal en bloque.

\begin{tabular}{ll}
\hline \multicolumn{1}{c}{ Donante $(\mathrm{n}=6)$} & Receptor $(\mathrm{n}=6)$ \\
\hline $\begin{array}{l}\text { Edad } \\
\text { Promedio: } 38 \text { meses }\end{array}$ & 29 años \\
Variación: 18 meses a 10 años & 13 años a 48 años \\
$\begin{array}{l}\text { Peso } \\
\text { Variación: } 15 \text { a } 22 \mathrm{~kg}\end{array}$ & 26 a $50 \mathrm{~kg}$ \\
Tiempo de isquemia fría & \\
Variación: 15 a 27 horas & \\
\hline
\end{tabular}

ureterales $\left({ }^{7,15,16,19,20}\right)$. Las investigaciones reportan que la masa nefronal reducida del injerto procedente de donante pediátrico es más susceptible a la injuria isquémica debido al tiempo de preservación y reperfusión, existiendo además alta predisposición al rechazo $\left({ }^{16}\right)$. Ante este panorama poco alentador en la sobrevida del injerto del donante pediátrico, surge la alternativa del trasplante renal en bloque con resultados excelentes $(3,4,17,21)$. En Latinoamérica, Chile reporta sus dos primeros trasplantes renales en bloque $\left({ }^{22}\right)$. Con este procedimiento, el receptor recibe doble número de nefronas que garantiza mejor la sobrevida del injerto a largo plazo $\left({ }^{3,15}\right)$. Los centros recomiendan realizar el trasplante renal en bloque sólo cuando los
Tabla 2. Monitoreo ecográfico del tamaño del injerto renal.

\begin{tabular}{lc}
\hline Dimensión longitudinal & Promedio (mm) \\
\hline Postoperatorio día 1 & 70 \\
Postoperatorio día 30 & 89 \\
Postoperatorio día 90 & 106 \\
\hline
\end{tabular}

donantes son menores de tres años o peso menor de 20 kilos $\left({ }^{3,15,23}\right)$ o tamaño renal menor de 5 $\mathrm{cm}$ de longitud $\left({ }^{24}\right)$.

Un reporte reciente menciona sobrevida del injerto en bloque de $84 \%$ al año de seguimiento $\left.{ }^{3}\right)$, duplicando el tamaño renal a los tres meses del postrasplante $\left({ }^{3}\right)$. La remoción en bloque de riñones pediátricos ofrece menor dificultad técnica y mejor flujo en el lecho arterial $\left({ }^{8,16}\right)$.

En esta comunicación, se comunica los resultados de seis pacientes que recibieron trasplante renal en bloque de donantes menores de 3 años de edad, siendo el donante más pequeño de 14 meses de edad y $11 \mathrm{~kg}$ de peso. Los seis injertos en bloque funcionaron inmediatamente después del trasplante y se adaptaron rápidamente a la demanda funcional de los receptores. En cinco de los seis receptores, los riñones incrementaron su volumen hasta en $55 \%$ al tercer mes postrasplante, lo que nos

Tabla 3. Complicaciones postrasplante renal en bloque.

\begin{tabular}{|c|c|c|}
\hline Paciente & Complicaciones & Resultados \\
\hline $41 \mathrm{M}$ & $\begin{array}{l}\text { Rechazo vascular severo P.O: } 10 \text { días } \\
\text { Trombosis venosa }\end{array}$ & Injertectomía bilateral P.O: 40 días \\
\hline $13 \mathrm{M}$ & $\begin{array}{l}\text { Rechazo crónico } \\
\text { Indisciplina a la medicación }\end{array}$ & $\begin{array}{l}\text { Pérdida del injerto } \\
\text { Reingreso a hemodiálisis P.O: } 2 \text { años }\end{array}$ \\
\hline $48 \mathrm{~F}$ & Estenosis ureteral unilateral P.O: 2 años & $\begin{array}{l}\text { Uropatía obstructiva unilateral } \\
\text { Riñón remanente creatinina } 2 \mathrm{mg} \% \\
\text { P.O: } 5 \text { años }\end{array}$ \\
\hline $39 \mathrm{M}$ & Ninguna & Creatinina 1,2 mg P.O: 4 años \\
\hline $15 \mathrm{M}$ & Ninguna & Creatinina 1,1 mg P.O: 3 años \\
\hline $22 \mathrm{M}$ & Ninguna & Creatinina 1,9 mg P.O: 8 meses \\
\hline
\end{tabular}

P.O: postoperatorio. 
indica que los primeros meses son particularmente importantes para su crecimiento y funcionalidad $\left({ }^{3,9}\right)$. Reportes previos confirman la rápida hipertrofia compensatoria de los riñones pediátricos cuando se les trasplanta en receptores adultos $\left({ }^{1,9,21,25}\right)$, a diferencia de lo que ocurre cuando se les trasplanta en receptor pediátrico, en los que no suceden cambios morfológicos tal como consignamos en uno de nuestros casos. Los centros de trasplante renal consideran que el trasplante renal en bloque de donante pediátrico realizado en receptor pediátrico, es el procedimiento más seguro, recomendable y fisiológico, reportando resultados de $100 \%$ de sobrevida del paciente y del injerto $\left({ }^{10,21,25}\right)$. Lamentablemente, los potenciales receptores pediátricos de nuestra lista de espera no tuvieron compatibilidad para la realización de este procedimiento.

En la evolución de estos trasplantes, no observamos proteinuria ni hipertensión arterial que indicara presencia de glomeruloesclerosis o alteración en la vascularización renal. Cuatro de los seis receptores cursaron con función renal adecuada a 4 años promedio de seguimiento, concordante con la sobrevida que reportan otros autores $\left({ }^{6,9,15}\right)$. La causa de la pérdida de uno de los casos de injerto en bloque fue secundario a trombosis venosa post rechazo vascular severo y la pérdida del otro caso fue por inobservancia a la medicación inmunosupresora a dos años de exitosa evolución. La trombosis vascular y el rechazo agudo del injerto son las causas más comunes de la pérdida temprana y tardía del injerto $\left({ }^{10}\right)$ y las complicaciones urológicas las menos comunes y de menor impacto en la sobrevida del injerto $\left({ }^{14}\right)$.

Esta experiencia nos enseña ser extremadamente cuidadosos en la selección de los receptores, vale decir adultos con bajo índice de masa corporal a fin de prevenir cambios compensatorios $\left({ }^{23,26}\right)$, además de poseer pocos factores de riesgo inmunológico, médico y social. La selección del receptor es elemento clave del éxito al usar estos riñones $\left({ }^{15}\right)$. Las investigaciones coinciden en señalar que la sobrevida del riñón de donante pediátrico menor de 2 años de edad es significativamente inferior cuando se los utiliza como trasplante renal simple $\left({ }^{15,25}\right)$. Otros estudios $\left({ }^{6,7,9,21}\right)$ confirman que dos años de edad parece ser la edad crítica para decidir el tipo de trasplante, recomendando que los riñones procedentes de donantes menores de 2 años de edad sean utilizados como trasplante renal en bloque y riñones de donantes mayores de 2 años como trasplante renal simple. Nosotros adoptamos la política de aceptar riñones de donantes pediátricos menores de 3 años de edad para utilizarlos como trasplante renal en bloque $\left({ }^{9}\right)$. El uso del micofenolato mofetil parece particularmente beneficioso en este tipo de receptores que son susceptibles al rechazo temprano ${ }^{1}$ y con el uso de la heparina de bajo peso molecular se logra minimizar el alto riesgo a la trombosis $\left({ }^{4,20}\right)$.

El presente estudio demuestra que es posible trasplantar exitosamente riñones de niños muy pequeños en receptores adultos. La doble masa nefronal implica duplicar la capacidad funcional y disminuir la hiperfiltración compensatoria $\left({ }^{8,23}\right)$. Nuestros resultados son alentadores y coinciden con lo obtenido en otros centros de alta actividad trasplantadora $\left({ }^{2}\right)$.

\section{REFERENCIAS BIBLIOGRÁFICAS}

1. Banowsky LH, Lackner J, Kothman R, Wright FH. Results of single kidneys from donors aged 9 to 60 months: Results in 144 adult recipients. Transplantation Proceedings. 1997;29:3271-3.

2. Lackmer JE, Wright FH, Banowsky LH. Long-term function of single pediatric kidneys less than 48 months of age transplanted into adult recipients compared with adult cadaveric and living-related trasplants. Transplantation Proceedings. 1997;29:3283-7.

3. Jordan ML, Shapiro R, Vivas CA, Scantlebury VP, Corry RJ. High-risk donors: Expanding donor criteria. Transplantation Proceedings. 1999;31:1401-3.

4. Abouna GM. Marginal donors: a viable solution for organ shortage. Transplatation Proceedings. 1997;29:2759-64.

5. Campistol JM. Trasplante renal en pacientes virus de la hepatitis C positivos. Nefrología. 2002;22 Suppl 5:62-6. 
6. Alshaibani K, Raza S, Almeshari K, Alfurayh O, Alahmadi I. Should pediatric kidneys be used in cadaveric kidney transplantation?. Transplantation Proceedings. 1998;30:3123-4.

7. Genyk Y, Wright R, Burrows L. Should pediatric donors younger than 2 years of age be used in kidney transplantation?. Transplantation Proceedings. 1997;29:3276-7.

8. Cransberg K, Van Gool JD, Davin JC, De Jong MCJW, Darby M. Pediatric renal transplantations in the Netherlands. Pediatric Transplantation. 2000;4:72-81.

9. Yagisawa T, Kam I, Chan L, Springer JW, Dunn S. Limitations of pediatric donor kidneys for transplantation. Clin Transplantation. 1998;12:557-62.

10. Geny Y, Burrows L, Knight RJ. Kidney transplantation utilizing donors from both age extremes. Transplantation Proceedings. 1997;29:3655-6.

11. Nezamudin NM, Adiku W, Farsi H, Al-Fayez S, Shaheen F. En bloc anencephalic cadaver donor renal transplantation. Transplantation Proceedings. 1989;20(1):1934-5.

12. Glasson J, Durham NC, Plows CW, Clarke OW. The use of anencephalic neonates as organ donors. JAMA. 1995;273(20): 1614-8.

13. Abbastiista AD, Vigevano F, Catenar G, Parisi F. Anencephalic neonates and diagnosis of death. Transplatation Proceedings. 1997;29:3634-5.

14. Geny Y, Knight R, de Csepel J, Burrows L. Successful transplantation of pediatric en bloc kidneys with bilateral double ureters. Traspl Int. 1999;12:281-2.

15. Hiramoto JS, Freire CE, Randall HR, Bretan PN, Tomlanovich S. Successful long-term outcomes using pediatric en bloc kidneys for transplantation. Am J Transplantation. 2002;2:337-42.

16. El-Sheik MFA, Gok MA, Buckley PE, Soomro N, Jacques BC. En bloc pediatric into adult recipients: The NewCastle Experience. Transplantation Proceedings. 2003;35:786-8.

17. Merkel FK. Transplantation of small en bloc kidneys including a horseshoe kidney from donors aged 2 to 60 months to adult recipients: a 13-year experience. Trasplantation Proceedings. 2001;33:3783-4.
18. Ramos E, Kasiske BL, Alexander SR, Danovich GM, Harmon WE. The evaluation of candidates for renal transplantation. Transplantation. 1994;57:490-7.

19. Strey C, Grotz W, Mutz C, Pisarsky O, Furtwaengler A. Graft survival and graft function of pediatric en bloc kidneys in paraaortal position. Transplantation. 2002;73(7):1095-9.

20. Portolés J, Marañes A, Prats D, Torrente J, Marrón B. Double renal transplant from infant donors. Transplantation. 1996;61:37-40.

21. BergMeijer JHLJ, Cransberg K, Nijman JM, Molenaar JC, Wolff ED. Functional adaptation of en bloc. Transplanted pediatric kidneys into pediatric recipients. Transplantation. 1994;58(5):623-5.

22. Troncoso P. Transplante renal en bloque de donantes pediátricos con interposición vascular iliaca (resumen). XVII Congreso de Trasplante de América Latina y el Caribe; 2003 Mayo; Cusco, Peru. p. 72.

23. Bretan PN, Freise C, Goldstein R, Osorio R, Lanovich ST. Selection strategies for successful utilization of less than $1,5 \mathrm{~kg}$ pediatic donor kidneys. Transplantation Proceedings. 1997;29:3274-5.

24. Berardinelli L. Long-term results of 211 single necrokidney transplantations from extreme-age donors. Why dual allograft? Transplantation Proceedings. 2001;33:3774-6.

25. Pugliese MR, Ridolfi L, Costa AN, Taddei S, Venturodi N. A comparison of pediatric and adult kidney donors for adult recipients. Transpl Int. 1999;12:122-6.

26. Bertram L, Kasiske J, Snyder, Gilbertson D. Inadequate donor size in cadaver kidney transplatation. J Am Soc Nephrol. 2002;13:2152-9.

Manuscrito recibido el 03 de noviembre de 2004 y aceptado para publicación el 15 marzo 2005.

Correspondencia: Dr. Pedro Méndez Chacón

Av. Los Tallanes G-34 Urb. La Capullana-Surco

Lima 33, Perú

Correo-e: zednem_ordep@yahoo.com 